\title{
SOLVABILITY OF MATRIX RICCATI EQUATIONS
}

\author{
V. V. Palin
}

UDC 517.9

\begin{abstract}
The paper is aimed at studying solvability conditions for the quadratic matrix Riccati equation that arises in connection with the Chapman-Enskog projection for the Cauchy problem and the mixed problem for moment approximations of kinetic equations. The structure of the matrix equation allows for the formulation of necessary and sufficient conditions for the existence of solutions in terms of eigenvectors and associated vectors of the coefficient matrix.
\end{abstract}

\section{Introduction}

This paper is aimed at studying solvability conditions for the matrix Riccati equation

$$
X A X+B X+X C+Q=0,
$$

which arises in connection with the Chapman-Enskog projection for the Cauchy problem and the mixed problem [2] for moment approximations of kinetic equations (see [1]). Here, the unknown $X$ is a complex matrix of size $(n-m) \times m$, and the matrices

$$
A \in \mathrm{M}_{m, m}(\mathbb{C}), \quad B \in \mathrm{M}_{n-m, m}(\mathbb{C}), \quad C \in \mathrm{M}_{m, n-m}(\mathbb{C}), \quad Q \in \mathrm{M}_{n-m, n-m}(\mathbb{C}), \quad n>m,
$$

are given, where $\mathrm{M}_{l, s}(\mathbb{C})$ is the space of complex matrices of size $l \times s$. This equation plays an important role in the study of linearized problems, in which case Eq. (1) has a special form, namely,

$$
\begin{gathered}
P_{21} \Lambda_{12} P_{21}-\Lambda_{22} P_{21}+P_{21} \Lambda_{11}-\Lambda_{21}=0, \\
A=\Lambda_{12}, \quad B=-\Lambda_{22}, \quad C=\Lambda_{11}, \quad Q=-\Lambda_{21},
\end{gathered}
$$

where the matrices $\Lambda_{11}, \Lambda_{12}, \Lambda_{21}$, and $\Lambda_{22}$ are blocks of the same matrix

$$
\Lambda=\left(\begin{array}{ll}
\Lambda_{11} & \Lambda_{12} \\
\Lambda_{21} & \Lambda_{22}
\end{array}\right)
$$

The structure (2) of the matrix equation (1) dictated by real physical problems allows for the statement of necessary and sufficient conditions for the solvability of Eq. (1) in terms of properties of eigenvectors and associated vectors of the matrix $\Lambda$.

Strange as it might seem, there is no reasonable theory for such a classical object as matrix equations! Gelfand wrote [3]: "The problem of solving quadratic equations for matrices seems to be absolutely classical. It would be natural that such a problem should have been at least formulated, or even solved, in the 19th century at the latest. Still, I asked many people about this problem, and they directed me to various sources, but nowhere could I find even a mention of this problem." Then Gelfand gave only one reference, namely, that to a result of D. B. Fuks. In addition, we can only make a reference to [4-7].

We give necessary and sufficient conditions for the solvability of Eq. (2). These conditions can be used to generalize the results of the papers mentioned above, but our interest in these topics is mostly connected with the Navier-Stokes problems of approximation of conservation laws with relaxation [1]. From the standpoint of the Chapman-Enskog projection, the most interesting case is that of a degenerate leading matrix $\Lambda_{12}$, i.e., $\operatorname{rank}\left(\Lambda_{12}\right)<\min \{n-m, m\}$, which is not allowed in [3,4].

Translated from Trudy Seminara imeni I. G. Petrovskogo, No. 27, Part II, pp. 281-300, 2009. 
S. I. Gelfand examples ([3]). Consider the quadratic Riccati equation for matrices

$$
X^{2}+B X+Q=0, \quad B, Q \in \mathrm{M}_{2}(\mathbb{C}) .
$$

The exotic nature of this classical object is demonstrated by the following two examples: the solutions of the quadratic matrix equation

$$
X^{2}=0
$$

are the matrices

$$
\left(\begin{array}{ll}
a & b \\
c & d
\end{array}\right)
$$

such that

$$
a d-b c=0, \quad a+d=0 .
$$

There are infinitely many such matrices and they form a two-dimensional cone in $\mathbb{C}^{4}$. On the other hand, the equation

$$
X^{2}=\left(\begin{array}{ll}
0 & 1 \\
0 & 0
\end{array}\right)
$$

has no solutions, since a matrix whose square has only zero eigenvalues itself must have only zero eigenvalues, which means that $X$ is a nilpotent matrix. But the square of any nilpotent $(2 \times 2)$-matrix is equal to zero.

Obviously, these are exceptions. But what then is the generic position? Equation (3) represents four second-order scalar equations. According to the Bezout theorem, there should be $2^{4}=16$ solutions in generic position. However, due to the matrix structure of the equation, the number of solutions turns out to be different. In [3], S. I. Gelfand made the following conjecture.

S. I. Gelfand hypothesis. "As far as I understand it, the answer to the question about these things, in general, may be the following. Still, I cannot prove everything, but if the classics of the British algebraic school of the middle or the end of the 19th century had not dealt with these problems, no one knows the answer. It seems that the situation is the following: there may be any number of solutions from 0 to 6 and also two types of infinity (a cone and a hyperboloid). Both infinities are two-dimensional."

In the present paper, we give an answer to the question about the number of solutions of the quadratic matrix equation of the form (2), depending on the parameters $m, n$, in most cases. In particular, the above Gelfand hypothesis turns out to be true.

The paper has the following structure. First, we describe how the problem of the existence of a projection for a linear system of partial differential equations is connected with a quadratic matrix equation of Riccati type. Then, we formulate and prove necessary and sufficient conditions for the existence of solutions and discuss the question about the number of solutions. The last part of the paper is dedicated to an important physical example, namely, the system of equations of phonon gas.

\section{Reduction of the Projection Problem to a Quadratic Matrix Equation}

Consider the Cauchy problem for a one-dimensional linear first-order hyperbolic system with constant coefficients with relaxation [1]

$$
\partial_{t} u+\mathcal{A} \partial_{x} u+B u=0
$$

Here, $x \in \mathbb{R}, u \in \mathbb{R}^{n}, A$ and $B$ are constant matrices. The Chapman-Enskog projection (see [8]) for system (4) is defined as follows:

$$
u=\Pi u_{c}=\left(u_{c}, \Pi_{21} u_{c}\right), \quad u_{c}=\left(u_{1}, \ldots, u_{m}, 0, \ldots, 0\right)^{\mathrm{T}},
$$

where $\Pi$ is a pseudodifferential matrix operator of zero order. Suppose that the matrix of the operator $\Pi$ describing the Chapman-Enskog projection into $m$ equations for system (4) has the form

$$
\Pi=\left(\begin{array}{ll}
\Pi_{11} & \Pi_{12} \\
\Pi_{21} & \Pi_{22}
\end{array}\right)
$$


where $\Pi_{11}=E_{m}$ is the identity matrix of size $m, \Pi_{22}=0_{n-m}$ is the zero square matrix of size $n-m$. Denote by $\Lambda$ the matrix $\mathcal{A} i \xi+B$. Let us split the matrix of the operator $\Lambda$ into blocks of the same size:

$$
\Lambda=\left(\begin{array}{ll}
\Lambda_{11} & \Lambda_{12} \\
\Lambda_{21} & \Lambda_{22}
\end{array}\right)
$$

Since $\Pi$ is a projector, we have

$$
\Pi \partial_{t} u_{c}+\mathcal{A} \Pi \partial_{x} u_{c}+B \Pi u_{c}=0
$$

Since $\Pi^{2}=\Pi$, we have

$$
\Pi \partial_{t} u_{c}+\Pi \mathcal{A} \Pi \partial_{x} u_{c}+\Pi B \Pi u_{c}=0 .
$$

Subtracting (6) from (5), we get

$$
(E-\Pi)\left(\mathcal{A} \partial_{x}+B\right) \Pi u_{c}=0
$$

Denote by $P$ the Fourier transform of the operator $\Pi$ with respect to $x$. The last relation, after the Fourier transformation in $x$, reads as follows:

$$
(E-P) \Lambda P v_{c}=0,
$$

i.e., $\Lambda P v_{c} \in \operatorname{Ker}(E-P)$. Representing any $v \in \operatorname{Ker}(E-P)$ in the form $v^{\mathrm{T}}=\left(v_{m}^{\mathrm{T}}, v_{n-m}^{\mathrm{T}}\right)$ with $v_{k} \in \mathbb{R}^{k}$, we have $v_{n-m}=P_{21} v_{m}$. Hence, we obtain a system of equations for $P_{21}$ that completely determines the projector $\Pi$ :

$$
P_{21}\left(\Lambda_{11}+\Lambda_{12} P_{21}\right)=\Lambda_{21}+\Lambda_{22} P_{21}
$$

Note that after opening the brackets and transferring all terms to the left-hand side, the above equation acquires the form (2), i.e., becomes a matrix equation of Riccati type.

\section{Necessary and Sufficient Conditions for the Existence of a Solution}

First, let us formulate and prove some theorems giving necessary and sufficient conditions for the solvability of Eq. (2) in the case $|\Lambda| \neq 0$.

Theorem 1. Suppose that $|\Lambda| \neq 0$ and the matrix $P$ has the form

$$
P=\left(\begin{array}{ll}
P_{11} & P_{12} \\
P_{21} & P_{22}
\end{array}\right)
$$

where $P_{11}$ is the identity matrix of size $m, P_{22}$ ia the zero square matrix of size $n-m, P_{12}$ is the zero matrix. Then the quadratic matrix equation (2) has a solution, if and only if there is a matrix $P$ that has the form described above and is a solution of the quadratic matrix equation

$$
(E-P) \Lambda P=0 .
$$

Indeed, suppose first that the matrix equation (2) has a solution. Then, constructing the matrix $P$ as described in the theorem and writing the product $(E-P) \Lambda P$ in block form, we see that the matrix $P$ is a solution of $(7)$.

Conversely, let $P$ be a solution of (7) with the above structure. Then, representing the matrix $M=(E-P) \Lambda P$ as blocks of the same size as $P$, we see that the structure of the matrix $P$ implies that the blocks $M_{11}, M_{12}, M_{22}$ consist of zeroes. The equation for the block $M_{21}$ coincides with (2) to within the sign, i.e., the matrix equation (2) is solvable.

Theorem 2. Let $|\Lambda| \neq 0$. Then the quadratic matrix equation (2) has a solution, if and only if there exist two solutions $X_{1}, X_{2}$ of the quadratic matrix equation

$$
X^{2}-\Lambda X=0
$$

such that

(1) $X_{1} e_{j}=0$ for all $j>m$;

(2) $e_{j}^{\mathrm{T}} X_{2}=e_{j}^{\mathrm{T}} \Lambda$ for all $j \leq m$;

(3) $\Lambda X_{2}=X_{1} \Lambda$. 
Suppose first that the matrix equation (2) has a solution. Therefore, Eq. (7), too, has a solution. Note that the matrix $P$ belongs to the class described above, if and only if $P e_{j}=0$ for all $j>m, e_{j}^{\mathrm{T}} P=e_{j}^{\mathrm{T}}$ for all $j \leq m$. Multiplying the matrix equation (7) by $\Lambda$ on the left and letting $X_{1}=\Lambda P$, we see that $X_{1}$ is a solution of equation (8) and it satisfies condition (1). Similarly, multiplying (7) by $\Lambda$ on the right and letting $X_{2}=P \Lambda$, we see that the matrix $X_{2}$ is a solution of (8) satisfying condition (2). Further, since $X_{1}=\Lambda P, X_{2}=P \Lambda$, it follows that condition (3) holds, too.

Suppose now that there exist two solutions $X_{1}, X_{2}$ of the matrix equation (8) and conditions (1)-(3) hold for these solutions. Let $P=\Lambda^{-1} X_{1}=X_{2} \Lambda^{-1}$. Then, conditions (1), (2) imply that the matrix $P$ has the desired structure. Next, inserting $X_{1}=\Lambda P$ into (8) and multiplying the resulting relation by $\Lambda^{-1}$ on the left, we see that $P$ is a solution of (7).

Theorem 3. Suppose that $|\Lambda| \neq 0$. Then the quadratic matrix equation (2) has a solution, if and only if there is a solution $X_{1}$ of the quadratic matrix equation (8) such that

(1) $X_{1} e_{j}=0$ for all $j>m$;

(2) $e_{j}^{\mathrm{T}} \Lambda^{-1} X_{1}=e_{j}^{\mathrm{T}}$ for all $j \leq m$.

Let $X_{2}=\Lambda^{-1} X_{1} \Lambda$. Obviously, $X_{2}$ is a solution of Eq. (8). Moreover, condition (2) implies that condition (2) of the previous theorem holds for the matrix $X_{2}$. Moreover, since $X_{2}=\Lambda^{-1} X_{1} \Lambda$, we have $\Lambda X_{2}=X_{1} \Lambda$, i.e., condition (3) of the preceding theorem holds, too.

In order to formulate the necessary and sufficient solvability conditions in another way, we first describe the general structure of solutions of the quadratic matrix equation (8).

Lemma 1. Let $\operatorname{det}(\Lambda) \neq 0$ and vectors $h_{1}, \ldots, h_{n}$ form the Jordan basis for the matrix $X$ which is a solution of Eq. (8). Then there is $K \geq 0$ such that the vectors $h_{1}, \ldots, h_{K}$ form part of the Jordan basis of the matrix $\Lambda$, with the order of association preserved (in the sense that if the vector $h_{j}$ satisfies the condition $X h_{j}=\lambda h_{j}+h_{j-1}$, then $\left.\Lambda h_{j}=\lambda h_{j}+h_{j-1}\right)$, and $h_{K+1}, \ldots, h_{n}$ are eigenvectors corresponding to the eigenvalue 0 .

Suppose that $v$ is an eigenvector of the unknown matrix $X$ corresponding to the eigenvalue $\lambda$. Then, multiplying the matrix equation (8) by $v$ on the right, we get

$$
\lambda^{2} v-\lambda \Lambda v=0,
$$

and since $v \neq 0$, it follows that

$$
\operatorname{det}\left(\lambda^{2} E-\lambda \Lambda\right)=0
$$

The last equation implies that each eigenvalue of the matrix $X$ must be either an eigenvalue of the matrix $\Lambda$ or zero. Moreover, for $\lambda \neq 0$, the equation for $v$ implies that $v$ is an eigenvector of the matrix $\Lambda$ corresponding to the same eigenvalue. If $\lambda=0$, then the corresponding vector $v$ is arbitrary.

Let us show that the matrix $X$ cannot have Jordan cells corresponding to the eigenvalue 0 and having the size greater than 1 . Let $v_{1}$ be an associated vector corresponding to the eigenvalue 0 . Then

$$
X v_{1}=\lambda v_{1}+v=v
$$

and, multiplying (8) by $v_{1}$ on the right, we obtain

$$
X v-\Lambda v=-\Lambda v=0,
$$

i.e., $v \in \operatorname{Ker}(\Lambda)$. But $\operatorname{det}(\Lambda) \neq 0$ by assumption, which means that we have come to a contradiction.

Now let $v_{1}$ be the first associated vector corresponding to a nonzero eigenvalue $\lambda$ of the matrix $X$, i.e., $X v_{1}=\lambda v_{1}+v, X v=\lambda v$. Then

$$
\lambda^{2} v_{1}+2 \lambda v-\lambda \Lambda v_{1}-\Lambda v=0
$$

and therefore,

$$
\Lambda v_{1}=\lambda v_{1}+v
$$


i.e., $v_{1}$ is the first associated vector also for the matrix $\Lambda$. Similarly, if $v_{k}$ is the $k$ th associated vector corresponding to the eigenvalue $\lambda$, for the matrix $X$, then it must be the $k$ th associated vector of the matrix $\Lambda$.

Lemma 2. Suppose that $\operatorname{det}(\Lambda) \neq 0, K \geq 0$ is an integer, and $X$ is a matrix whose Jordan basis consists of vectors $h_{1}, \ldots, h_{n}$, where $h_{1}, \ldots, h_{K}$ form part of the Jordan basis of the matrix $\Lambda$ with the order of association preserved, and $h_{K+1}, \ldots, h_{n}$ are eigenvectors corresponding to the eigenvalue 0 . Then $X$ is a solution of the matrix equation (8).

Let $M$ be the matrix whose columns coincide with the vectors $h_{j}$, and let $J(X)$ be the Jordan form of the matrix $X$. Then, $X=M J(X) M^{-1}$, and after its insertion into Eq. (8), the left-hand side takes the form

$$
M J^{2}(X) M^{-1}-\Lambda M J(X) M^{-1} .
$$

Set $f(v)=\left(M J^{2}(X) M^{-1}-\Lambda M J(X) M^{-1}\right) v$. Note that the function $f(v)$ is linear. Moreover, $f\left(h_{j}\right)=0$ for any $j=1, \ldots, n$. Thus, $M J^{2}(X) M^{-1}-\Lambda M J(X) M^{-1}=0$, i.e., the matrix $X=M J(X) M^{-1}$ is a solution of Eq. (8).

Let us give another geometrical statement of necessary and sufficient conditions for the existence of a solution of the quadratic matrix equation (2).

Theorem 4. Let $|\Lambda| \neq 0$. Suppose that there are vectors $v_{1}, \ldots, v_{m}$ such that:

(1) $V=\operatorname{Lin}\left\{v_{j}\right\}_{1}^{m}$ is an eigenspace of the matrix $\Lambda$, i.e., $\Lambda V=V$;

(2) the vectors $v_{1}, \ldots, v_{m}, e_{m+1}, \ldots, e_{n}$ form a basis.

Then the quadratic matrix equation (2) has a solution, and the converse is also true.

\section{The Case of Generic Position}

Now suppose that $|\Lambda|=0$ and $V_{0}$ is the linear hull of all eigen and associated vectors of $\Lambda$ corresponding to the eigenvalue 0 .

Definition 1. We say that problem (2) is in generic position if

$$
\forall v \in V_{0}: \quad e_{j}^{\mathrm{T}} v=0 \forall j>m .
$$

First, let $L=\operatorname{dim} V_{0}<m$. In this case, we describe a method that would allow us to reduce the problem in generic position to the non-singular case considered above. To that end, we need some auxiliary lemmas.

Lemma 3. For any square matrix $A$, there are two square matrices $I, M$ such that:

(1) $A=M I=I M$;

(2) $M$ is invertible;

(3) $I v=A v, \forall v \in V_{0}$;

(4) $I v=v$ for any $v$ orthogonal to $V_{0}$.

Obviously, in order to define a matrix, it suffices to specify its action on some basis. Let us specify the action of the matrices $M, I$ on the Jordan basis of the matrix $A$.

(1) If a vector $v$ of the Jordan basis corresponds to the eigenvalue 0 , then let $M v=v, I v=A v$.

(2) If a vector $v$ of the Jordan basis corresponds to a non-zero eigenvalue, then let $M v=A v, I v=v$.

It is easy to check that the matrices $M, I$ defined in this way satisfy all conditions of the lemma.

Lemma 4. Let (2) be a problem in generic position and let $m>\operatorname{dim} V_{0}$. Then, there is a matrix $C$ such that there exist two matrices $M, I$ satisfying the following conditions:

(1) $C^{-1} \Lambda C=I M=M I$; 
(2) $C$ is a block-diagonal matrix:

$$
C=\left(\begin{array}{cc}
C_{11} & 0 \\
0 & E
\end{array}\right)
$$

where $C_{11}$ is a square matrix of size $m$;

(3) the matrices $M, I$ satisfy the conditions of the previous lemma;

(4) I is a block-diagonal matrix:

$$
I=\left(\begin{array}{ccc}
I_{1} & 0 & 0 \\
0 & I_{2} & 0 \\
0 & 0 & E
\end{array}\right)
$$

where $I_{1}$ is a square nilpotent matrix of size $\operatorname{dim} V_{0}$, and $I_{2}$ is the identity matrix of size $m-\operatorname{dim} V_{0}$;

(5) if the matrix $M$ is split into blocks of the same size as $I$, then $M_{11}=E, M_{12}=0, M_{13}=0$, $M_{21}=0$, and $M_{31}=0$.

First, let us represent the matrix $\Lambda$ in the form $\Lambda=I^{\prime} M^{\prime}$, where $I^{\prime}$ and $M^{\prime}$ are matrices from the preceding lemma. Let $C$ be a matrix such that $C^{-1} I^{\prime} C$ is the Jordan normal form of the matrix $I$, with its first cells corresponding to the eigenvalue 0 . Then the matrix $I$ has the desired structure. Since we have a problem in generic position, it follows that among all matrices $C$ that reduce $I^{\prime}$ to the Jordan normal form, there is a matrix satisfying condition (2).

It remains to show that condition (5) holds for the matrix $C^{-1} M C$. By the preceding lemma, we have $M v=v$ for any $v \in V_{0}$. On the other hand, if the matrix $I$ has the desired structure, then $V_{0}=\operatorname{Lin}\left\{e_{1}, \ldots, e_{L}\right\}, L<m$. Hence, we get $M e_{j}=e_{j}$ for all $j \leq L$. Therefore, $M_{21}=0$ and $M_{31}=0$. Let $V_{1}$ be a linear subspace such that for all $v \in V_{0}, w \in V_{1}$, we have $v^{\mathrm{T}} w=0$, $\operatorname{dim} V_{0}+\operatorname{dim} V_{1}=n$. Let us show that for all $u \in V_{1}$, we have $M u \in V_{1}$. Note that the subspace $V_{1}$ is invariant for $\Lambda$, i.e., $\Lambda V_{1}=V_{1}$. Therefore, it is invariant for $M$ due to the construction of $M$. It follows that $M_{12}=0$ and $M_{13}=0$.

Note that an analogue of this lemma holds for the case of $L=\operatorname{dim} V_{0}=m$. In this case, the blocks that should have size 0 are just absent.

Lemma 5. Let $C$ be a block-diagonal matrix of the same form as in the preceding lemma. Then, the solution of the quadratic matrix equation

$$
\left(E-P^{\prime}\right) C \Lambda C^{-1} P^{\prime}=0
$$

is related to the solution $P$ of the matrix equation (7) by $P_{11}^{\prime}=P_{11}, P_{21}^{\prime}=P_{21} C_{11}^{-1}, P_{12}^{\prime}=C_{11} P_{12}$, and $P_{22}^{\prime}=P_{22}$.

Note that if the matrix $P$ is a solution of (7), then the matrix $P^{\prime}=C P C^{-1}$ is a solution of (9). Hence, taking into account the block structure of the matrix $C$, we obtain the desired result.

Next, we describe how the problem in generic position can be reduced to the case considered above. Note that Theorem 1 holds also in the case of $|\Lambda|=0$. Consider the matrix $P$ which is the solution of (7) described in Theorem 1. Let us split $P$ into blocks of the same size as the blocks of the matrix $I$ in Lemma 4:

$$
P=\left(\begin{array}{ccc}
E & 0 & 0 \\
0 & E & 0 \\
\Pi_{31} & \Pi_{32} & 0
\end{array}\right) .
$$

By Lemma 5, if $P$ has the above structure, then the matrix $P^{\prime}$ has the same structure:

$$
P^{\prime}=\left(\begin{array}{ccc}
E & 0 & 0 \\
0 & E & 0 \\
\Pi_{31}^{\prime} & \Pi_{32}^{\prime} & 0
\end{array}\right) .
$$


Theorem 5. Let (2) be a problem in generic position and $\operatorname{dim} V_{0}<m$. This problem has a solution, if and only if the problem of the same form for the matrix

$$
\Lambda^{\prime}=\left(\begin{array}{ll}
M_{22} & M_{23} \\
M_{32} & M_{33}
\end{array}\right)
$$

has a solution, where $M$ is the matrix described in Lemma 4.

Let us represent the matrix $\Lambda$ in the form $\Lambda=C^{-1} I M C$, according to Lemma 4. Since the solvability of (2) is equivalent to that of (7), consider the equation

$$
(E-P) \Lambda P=0 .
$$

Inserting the expression for $\Lambda$ into the latter, we get

$$
(E-P) C^{-1} I M C P=0 .
$$

This equation is obviously equivalent to

$$
\left(E-P^{\prime}\right) I M P^{\prime}=0
$$

where $P^{\prime}$ is the matrix described in Lemma 5. Writing the last equation block-wise, we obtain a system of two equations equivalent to the original equation (2):

$$
\begin{gathered}
\Pi_{31}^{\prime} I_{1}+\Pi_{32}^{\prime} M_{23} \Pi_{31}^{\prime}-M_{33} \Pi_{31}^{\prime}=0, \\
\Pi_{32}^{\prime} M_{23} \Pi_{32}^{\prime}-M_{33} \Pi_{32}^{\prime}+\Pi_{32}^{\prime} M_{22}-M_{32}=0 .
\end{gathered}
$$

Not that the second equation of this system is an equation of the form (2) for the matrix $\Lambda^{\prime}$ specified in Theorem 5, while the first equation always has the solution $\Pi_{31}^{\prime}=0$. The theorem is proved.

It remains to note that $\left|\Lambda^{\prime}\right|=|M| \neq 0$, and thus, we have described a method for reducing problem (2) in generic position (for $\operatorname{dim} V_{0}<m$ ) to the non-singular case.

Let us say a few words about the dimension of the space of solutions of the problem in generic position, $\operatorname{dim} V_{0}<m$. In the non-singular case, the number of solutions of problem (2) is determined by the Jordan form of $\Lambda$, and therefore, the number of solutions in the case of generic position can be "greater," if and only if the equation

$$
\Pi_{31}^{\prime} I_{1}+\Pi_{32}^{\prime} M_{23} \Pi_{31}^{\prime}-M_{33} \Pi_{31}^{\prime}=0
$$

has infinitely many solutions, in which case the dimension of the "additional" space of solutions of this equation and that of the original problem (2) coincide.

Now let $\operatorname{dim} V_{0}=m$. Then, reasoning as above, we can reduce the original problem (2) to the quadratic matrix equation

$$
\left(\begin{array}{cc}
0 & 0 \\
\Pi_{21}^{\prime} & -E
\end{array}\right)\left(\begin{array}{cc}
I_{1} & 0 \\
0 & E
\end{array}\right)\left(\begin{array}{cc}
E & 0 \\
0 & M_{22}
\end{array}\right)\left(\begin{array}{cc}
E & 0 \\
\Pi_{21}^{\prime} & 0
\end{array}\right)=0
$$

which brings us to the following equation for $\Pi_{21}^{\prime}$ :

$$
\Pi_{21}^{\prime} I_{1}-M_{22} \Pi_{21}^{\prime}=0 .
$$

Obviously, the last equation is always solvable, and the dimension of the space of its solutions, as above, coincides with the space of solutions of the original problem (2).

\section{Number of Solutions in the Case of Generic Position}

Lemma 6. Let $A$ be an invertible matrix and $B$ a nilpotent matrix. Then the matrix equation $A X-X B=0$ has only one solution, which is identically equal to zero. 
Let $X$ be a solution of the equation $A X-X B=0$. Since $B$ is nilpotent, there is $s$ such that $B^{s}=0$. Let us multiply the identity $A X-X B=0$ by $B^{s-1}$ on the right. We get $A X B^{s-1}=0$, and therefore, using the invertibility of $A$, we find that $X B^{s-1}=0$. Further, multiplying the same identity by $B^{s-2}$ on the right, we obtain $X B^{s-2}=0$, etc. Thus, in finitely many steps, we find that $X=0$.

The above lemma implies the uniqueness of a solution of Eq. (2) for $L=m$. Now let $L<m$.

Lemma 7. Let $P_{21}$ be a solution of Eq. (2), $|\Lambda| \neq 0$. Then, the matrix $\Lambda_{22}-P_{21} \Lambda_{12}$ is invertible.

Let $K=(E-P) \Lambda+\lambda P$, where the matrix $P$ is the projector corresponding to the block $P_{21}$. Let

$$
\mathcal{L}_{c}=c \Lambda^{-1} P+E, \quad \mathcal{R}_{c}=c(E-P) \Lambda^{-1}+E .
$$

It is easy to see that

$$
\mathcal{L}_{c} K \mathcal{R}_{c}=K+c E .
$$

Let us chose $c$ such that $\operatorname{det}(K+c E) \neq 0$. Then the determinant of the matrix on the left-hand side does not vanish, too. Since the determinant of a product of matrices is equal to the product of the corresponding determinants, it follows that $|K| \neq 0$.

Note that

$$
K=\left(\begin{array}{cc}
\Lambda_{11}+\Lambda_{12} P_{21} & 0 \\
2 \Lambda_{21}+\Lambda_{22} P_{21}-P_{21} \Lambda_{11} & \Lambda_{22}-P_{21} \Lambda_{12}
\end{array}\right)
$$

and therefore,

$$
|K|=\left|\Lambda_{11}+\Lambda_{12} P_{21}\right|\left|\Lambda_{22}-\Lambda_{21} P_{12}\right| \neq 0,
$$

which implies the statement of the lemma.

Since the matrix equation (2) for $L<m$ can be reduced to the system

$$
\begin{gathered}
\Pi_{31}^{\prime} I_{1}+\Pi_{32}^{\prime} M_{23} \Pi_{31}^{\prime}-M_{33} \Pi_{31}^{\prime}=0, \\
\Pi_{32}^{\prime} M_{23} \Pi_{32}^{\prime}-M_{33} \Pi_{32}^{\prime}+\Pi_{32}^{\prime} M_{22}-M_{32}=0,
\end{gathered}
$$

where the matrix

$$
\Lambda^{\prime}=\left(\begin{array}{ll}
M_{22} & M_{23} \\
M_{32} & M_{33}
\end{array}\right)
$$

is invertible and the matrix $I_{1}$ is nilpotent, it follows from the previous two lemmas that the space of solutions of Eq. (2) in this case has the same dimension and the space of solutions of problem (2) with the matrix $\Lambda^{\prime}$.

\section{Example: Projection Problem for Phonon Gas Equations}

As an example, consider the one-dimensional model of phonon gas $[2,8]$

$$
\begin{gathered}
\partial_{t} e+\partial_{x} p=0, \\
\partial_{t} p+\alpha_{1} \partial_{x} e+\partial_{x} N+q p=0, \\
\partial_{t} N+\alpha_{2} \partial_{x} p+\partial_{x} N_{1}+N=0, \\
\cdots \\
\partial_{t} N_{n-1}+\alpha_{n+1} \partial_{x} N_{n-2}+\partial_{x} N_{n}+N_{n-1}=0, \\
\partial_{t} N_{n}+\alpha_{n+2} \partial_{x} N_{n-1}+N_{n}=0, \\
\alpha_{j}=\frac{j^{2}}{4 j^{2}-1}, \quad q \in(0,1) .
\end{gathered}
$$

For this system, we prove the existence of irreducible projections $2 k+1 \rightarrow 1$ and $2 k+1 \rightarrow 2 k$ for odd $n=2 k+1$, and the existence of an irreducible projection $2 k \rightarrow 2$ for even $n=2 k$. The matrix 
$\Lambda=\left(\lambda_{i j}\right)_{i, j=1}^{n}$ for system (10) can be described as follows:

$$
\begin{gathered}
\lambda_{11}=0, \quad \lambda_{22}=q, \\
\lambda_{j j}=1 \forall j>2, \quad \lambda_{j k}=0 \forall j, k:|j-k|>1, \\
\lambda_{j, j+1}=i \xi \forall j=1, \ldots, n-1 ; \quad \lambda_{j+1, j}=i \alpha_{j} \xi \forall j=1, \ldots, n-1 .
\end{gathered}
$$

Projections $2 \boldsymbol{k}+\mathbf{1} \rightarrow \mathbf{1}$ and $\mathbf{2 k}+\mathbf{1} \rightarrow \mathbf{2} \boldsymbol{k}$. Let us show that if $n=2 k+1, m=1$ or $m=2 k$, then the projection exists always. It is easy to see that the determinant $|\Lambda|$ does not vanish for any $|\xi|>0$. Let $\xi=0$. For the matrix $\Lambda$, the subspace $V_{0}$ coincides with $\operatorname{Lin}\left\{e_{1}\right\}$, and therefore, $\Lambda$ satisfies all conditions of the generic position both for $m=1$ and $m=2 k$. Hence obviously follows the existence of the projection into $m$ equations for $\xi=0$ (for $m=1$ this is the case in which $m=\operatorname{dim} V_{0}$, and the projection always exists; for $m=2 k$ the existence of the projection is established by explicit calculations).

Now let $|\xi| \neq 0$. Since $n=2 k+1$, the matrix $\Lambda$ always has an eigenvalue that corresponds to a real solution after quantization.

Definition 2. We say that system (2) admits quantization, if it is possible to pass from system (2) (its solutions are complex-valued functions of the parameter $\xi$, in general) to an equivalent system of quadratic equations whose solutions are real-valued functions of $|\xi|^{2}$.

The said eigenvalue corresponds to an eigenvector $v$ that also brings us to real projectors after the quantization procedure. Moreover, as shown above, we have $e_{1}^{\mathrm{T}} v \neq 0$, and therefore, $v, e_{2}, \ldots, e_{n}$ is a basis. Hence we obtain the existence of the projection into 1 equation. Besides, the subspace $W$, obtained as the linear hull of all vectors of the Jordan basis of the matrix $\Lambda$, apart from the vector $v$, also leads to real projectors after quantization. Let us show that $e_{n} \notin W$, which would imply the existence of the projection into $2 k$ equations.

We establish a more general fact: If $W_{r}$ is an invariant subspace of the matrix $\Lambda$ and its dimension $r$ satisfies the condition $r \leq n-1$, then the subspaces $W_{r}$ and $\operatorname{Lin}\left\{e_{r+1}, \ldots, e_{n}\right\}$ have no common points but zero. The proof of this statement can be split into three parts corresponding to the following three lemmas.

Lemma 8. Let $w_{1}, \ldots, w_{r}$ be a basis of $W_{r}$ that forms part of the Jordan basis of the matrix $\Lambda$. Suppose also that all $w_{j}$ correspond to the same eigenvalue $\lambda$ and $\Lambda w_{1}=\lambda w_{1}, \Lambda w_{k+1}=\lambda w_{k+1}+w_{k}$. Then the subspaces $W_{r}$ and $\operatorname{Lin}\left\{e_{r+1}, \ldots, e_{n}\right\}$ have no common points but zero.

Let $c_{1} w_{1}+\cdots+c_{r} w_{r} \in \operatorname{Lin}\left\{e_{r+1}, \ldots, e_{n}\right\}$. Then for any $k<r$, we have

$$
\Lambda^{k}\left(c_{1} w_{1}+\cdots+c_{r} w_{r}\right) \in \operatorname{Lin}\left\{e_{r+1-k}, \ldots, e_{n}\right\},
$$

and therefore,

$$
e_{1}^{\mathrm{T}} \Lambda^{k}\left(c_{1} w_{1}+\cdots+c_{r} w_{r}\right)=0 \quad \forall k: 0 \leq k \leq r-1 .
$$

Relations (11) form a homogeneous linear system of equations for the unknown coefficients $c_{j}$. Let us show that the determinant of this system differs from zero. To that end, we first write out formulas for the elements $a_{k j}$ of the matrix of this system. Let $a_{k j}=e_{1}^{\mathrm{T}} \Lambda^{k-1} w_{j}$. By induction, it is easy to show that

$$
a_{k j}=\sum_{i=0}^{j-1} C_{k-1}^{i} \lambda^{k-1-i} a_{1, j-i} .
$$

Indeed, for $k=1$ and $j=1$, this formula is valid. Assuming that it holds for $a_{k, j}$, we obtain

$$
\begin{aligned}
a_{k+1, j}=e_{1}^{\mathrm{T}} \Lambda^{k} w_{j}=e_{1}^{\mathrm{T}} \lambda\left(\Lambda^{k-1} w_{j}\right)=e_{1}^{\mathrm{T}} \Lambda\left(\sum_{i=0}^{j-1} C_{k-1}^{i} \lambda^{k-1-i} w_{j-i}\right) & \\
& =e_{1}^{\mathrm{T}} \sum_{i=0}^{j-1}\left(C_{k-1}^{i}+C_{k-1}^{i-1}\right) \lambda^{k-i} w_{j-i}=e_{1}^{\mathrm{T}} \sum_{i=0}^{j-1} C_{k}^{i} \lambda^{k-i} w_{j-i}
\end{aligned}
$$


as desired. Next, we make the following linear transformation of the rows of the matrix of system (11):

$$
a_{k, j}^{\prime}=\sum_{s=0}^{k-1} C_{k-1}^{s}(-\lambda)^{s} a_{k-s, j} .
$$

Using the explicit formula for $a_{k, j}$, we find the result of the transformation (12) for $k \geq j$ :

$$
\begin{aligned}
a_{k, j}^{\prime}=\sum_{s=0}^{k-1} C_{k-1}^{s}(-1)^{s} \lambda^{s} a_{k-s, j}=\sum_{s=0}^{k-1} \sum_{i=0}^{j-1}(-1)^{s} C_{k-1}^{s} \lambda^{s} C_{k-s-1}^{i} \lambda^{k-s-1-i} a_{1, j-i} \\
=\sum_{s=0}^{k-1} \sum_{i=0}^{j-1}(-1)^{s} C_{k-1}^{s} C_{k-s-1}^{i} \lambda^{k-1-i} a_{1, j-i}=\sum_{i=0}^{j-1}\left(\sum_{s=0}^{k-1}(-1)^{s} C_{k-1}^{s} C_{k-s-1}^{i}\right) \lambda^{k-1-i} a_{1, j-i} .
\end{aligned}
$$

Let us calculate the sum in brackets. Note that $C_{n}^{m}=0$ for $m>n$. Thus, it can be assumed that the summation is up to $k-i-1$. Therefore,

$$
\begin{gathered}
\sum_{s=0}^{k-i-1}(-1)^{s} C_{k-1}^{s} C_{k-s-1}^{i}=\sum_{s=0}^{k-i-1}(-1)^{s} \frac{(k-1) !}{s !(k-s-1) !} \frac{(k-s-1) !}{i !(k-s-i-1) !}=\sum_{s=0}^{k-i-1}(-1)^{s} \frac{(k-1) !}{i ! s !(k-i-s-1) !} \\
=\sum_{s=0}^{k-i-1}(-1)^{s} \frac{(k-1) !(k-i-1) !}{s ! i !(k-i-1) !(k-s-i-1) !}=\sum_{s=0}^{k-i-1}(-1)^{s} C_{k-1}^{i} C_{k-1-i}^{s}=C_{k-1}^{i} \sum_{s=0}^{k-1-i}(-1)^{s} C_{k-1-i}^{s} .
\end{gathered}
$$

The last sum is equal to zero for $k-1>i$, and for $k-1=i$ it is equal to 1 . It follows that

$$
a_{k, j}^{\prime}=0, \quad k>j ; \quad a_{k, j}^{\prime}=a_{1,1}, \quad k=j .
$$

Thus, the transformation (12) reduces the matrix of system (11) to upper triangular form, with the main diagonal elements coinciding with $a_{1,1}=e_{1}^{\mathrm{T}} w_{1} \neq 0$, since $w_{1}$ is an eigenvector of the matrix $\Lambda$ and $\lambda \neq 0$. It follows that the matrix of system (11) has a nonzero determinant, and therefore, the system has only the trivial solution, i.e., $c_{j}=0$ for all $j=1, \ldots, r$. The lemma is proved.

Further, to prove our hypothesis, we use induction with respect to the dimension $r$.

Lemma 9. Suppose that the subspaces $W_{r}$ and $\operatorname{Lin}\left\{e_{r+1}, \ldots, e_{n}\right\}$ have no common points but zero, $r \geq 1$, $W_{r+1}$ is also an invariant subspace of the matrix $\Lambda$ such that for all $u \in W_{r+1}$ we have $u=v+w$, where $w \in W_{r}, v$ is an eigenvector of $\Lambda$ corresponding to the eigenvalue $\lambda$. Then the subspaces $W_{r+1}$ and $\operatorname{Lin}\left\{e_{r+2}, \ldots, e_{n}\right\}$ have no common points but zero.

Since $W_{r}$ and $W_{r+1}$ are invariant subspaces and each eigenvalue $\lambda$ of the matrix $\Lambda$ corresponds to a single eigenvector, it follows that the matrix $\Lambda-\lambda E$ is invertible on $W_{r}$. Let $u=c_{1} v+c_{2} w \in$ $\operatorname{Lin}\left\{e_{r+2}, \ldots, e_{n}\right\}, w \in W_{r}$. Then $\Lambda u \in \operatorname{Lin}\left\{e_{r+1}, \ldots, e_{n}\right\}$. Thus, for any $p \leq r$, we have $e_{p}^{\mathrm{T}}\left(c_{1} v+c_{2} w\right)=0$, $e_{p}^{\mathrm{T}} \Lambda\left(c_{1} v+c_{2} w\right)=0$. Hence, using the relation $\Lambda v=\lambda v$, we find that

$$
c_{2} e_{p}^{\mathrm{T}}(\Lambda-\lambda E) w=0 \quad \forall p \leq r,
$$

i.e., either $(\Lambda-\lambda E) w \in \operatorname{Lin}\left\{e_{r+1}, \ldots, e_{n}\right\}$ or $c_{2}=0$. But the matrix $\Lambda-\lambda E$ is invertible on $W_{r}$, and therefore, using the assumptions, we find that in the first case, $w=0$. Then $v \in\left\{e_{r+2}, \ldots, e_{n}\right\}$, which is impossible, since $v$ is an eigenvector. If $c_{2}=0$, then the relation $c_{1} e_{1}^{\mathrm{T}} v=0$ implies that $c_{1}=0$. The lemma is proved.

Lemma 10. Suppose that for all $t, 1 \leq t \leq r$, the subspaces $W_{t}$ and $\operatorname{Lin}\left\{e_{t+1}, \ldots, e_{n}\right\}$ have no points in common but zero. Let $W_{r+1}$ be also an invariant subspace of the matrix $\Lambda$ such that for all $u \in W_{r+1}$, we have $u=v+w$, where $w \in W_{r}$ and $v$ is an associated vector of the matrix $\Lambda$ corresponding to the eigenvalue $\lambda$. Then the subspaces $W_{r+1}$ and $\operatorname{Lin}\left\{e_{r+2}, \ldots, e_{n}\right\}$ have no points in common but zero. 
Let $w_{1}, \ldots, w_{p}$ form part of a basis in $W_{r+1}$ such that $w_{p}=v$ and any $u \in W_{r+1}$ can be represented in the form $u=w+c_{1} w_{1}+\cdots+c_{p} w_{p}$, where $w \in W_{r+1-p}$. On the subspace $W_{r+1-p}$, the matrix $\Lambda-\lambda E$ is invertible. Moreover, let $\Lambda w_{1}=\lambda w_{1}, \Lambda w_{k+1}=\lambda w_{k+1}+w_{k}$. Suppose that $u=c_{1} w_{1}+\cdots+c_{p} w_{p}+c_{p+1} w \in$ $\operatorname{Lin}\left\{e_{r+2}, \ldots, e_{n}\right\}$. Then, we obtain the following system of equations for the coefficients $c_{j}$ :

$$
e_{l}^{\mathrm{T}} \Lambda^{k}\left(c_{1} w_{1}+\cdots+c_{p} w_{p}+c_{p+1} w\right)=0, \quad 0 \leq k \leq p,
$$

where $l \in 1, \ldots, r+1-p$. Note that this system has the same form as (11). Let us apply the transformation (12) to the rows of the matrix of this system. Then the matrix composed of the elements $a_{k, j}^{\prime}$ is upper triangular and all its diagonal elements but one are equal to $a_{1,1}$. The last diagonal element is equal to

$$
a_{p+1, p+1}^{\prime}=\sum_{s=0}^{p} C_{p}^{s}(-\lambda)^{s} a_{p-s, p}=\sum_{s=0}^{p} C_{p}^{s}(-\lambda)^{s} e_{l}^{\mathrm{T}} \Lambda^{p-s} w=e_{l}^{\mathrm{T}}(\Lambda-\lambda E)^{p} w .
$$

Note that $w \neq 0$, since otherwise we would have a contradiction with the above lemma about a single Jordan cell. Since $w \in W_{r+1-p}$, using our assumptions, we can find $l \leq r+1-p$ such that $e_{l}^{\mathrm{T}}(\Lambda-\lambda E)^{p} w \neq 0$, since the matrix $\Lambda-\lambda E$ maps $W_{r+1-p}$ into itself. Therefore, there is $l$ such that the determinant of the matrix of the system for $c_{j}$ differs from zero. This means that all $c_{j}$ are equal to zero. The lemma is proved.

Projection $2 \boldsymbol{k} \rightarrow \mathbf{2}$. Consider the case of even $n=2 k$. Let us establish the existence of a projection from $n=2 k$ to $m=2$. To that end, we note that $|\Lambda| \neq 0$ for all $|\xi|>0$. If $\xi=0$, then the matrix $\Lambda$ satisfies all conditions of the generic position. Moreover, for $\xi=0$ the matrix $\Lambda$ is diagonal, and this obviously implies that there exists a projection into two equations for $\xi=0$.

Now let $\xi \neq 0$. If $v$ is an eigenvector of the matrix $\Lambda$ corresponding to the eigenvalue $\mu \neq 0$, then $e_{1}^{\mathrm{T}} v \neq 0$. Indeed, assume the contrary. From the structure of the matrix $\Lambda$ we see that $\mu e_{1}^{\mathrm{T}} v=i \xi e_{2}^{\mathrm{T}} v$, and therefore, $e_{2}^{\mathrm{T}} v=0$. This relation, in combination with

$$
\mu e_{2}^{\mathrm{T}} v=\alpha_{1} i \xi e_{1}^{\mathrm{T}} v+q e_{2}^{\mathrm{T}} v+i \xi e_{3}^{\mathrm{T}} v
$$

implies that $e_{3}^{\mathrm{T}} v=0$, etc. Thus, we find that $v=0$, which is impossible. Therefore, any eigenvector of the matrix $\Lambda$ corresponding to a nonzero eigenvalue has its first coordinate different from zero.

Now let $v_{1}$ and $v_{2}$ be two eigenvectors corresponding to two distinct nonzero eigenvalues $\mu_{1}$ and $\mu_{2}$, respectively. Let us show that no linear combination of $v_{1}$ and $v_{2}$ belongs to the linear hull of the vectors $\left\{e_{3}, \ldots, e_{n}\right\}$. Assume the contrary. Let $c_{1} v_{1}+c_{2} v_{2} \in \operatorname{Lin}\left\{e_{3}, \ldots, e_{n}\right\}$. Then $\Lambda\left(c_{1} v_{1}+c_{2} v_{2}\right)=$ $\mu_{1} c_{1} v_{1}+\mu_{2} c_{2} v_{2} \in \operatorname{Lin}\left\{e_{2}, \ldots, e_{n}\right\}$. Hence, we obtain a system of two equations for $c_{j}$ with two unknown quantities:

$$
\begin{gathered}
\left(e_{1}^{\mathrm{T}} v_{1}\right) c_{1}+\left(e_{1}^{\mathrm{T}} v_{2}\right) c_{2}=0, \\
\mu_{1}\left(e_{1}^{\mathrm{T}} v_{1}\right) c_{1}+\mu_{2}\left(e_{1}^{\mathrm{T}} v_{2}\right) c_{2}=0 .
\end{gathered}
$$

Since $\mu_{1} \neq \mu_{2}$, this system has a nonzero determinant, and therefore, $c_{1}=c_{2}=0$. This is a contradiction. Thus, if $v_{1}$ and $v_{2}$ are two eigenvectors corresponding to two distinct eigenvalues $\mu_{1}$ and $\mu_{2}$, then their linear combinations cannot belong to the linear hull of the vectors $\left\{e_{3}, \ldots, e_{n}\right\}$.

Next, we note that the structure of the matrix $\Lambda$ implies that any eigenvalue $\mu$ corresponds to precisely one eigenvector (to within a coefficient). Indeed, let $v$ correspond to the eigenvalue $\mu$ and $v=\left(v_{1}, \ldots, v_{n}\right)^{\mathrm{T}}$. Then, the explicit form of the matrix $\Lambda$ shows that $v_{2}=\frac{\mu}{i \xi} v_{1}, v_{3}=\frac{(\mu-q) v_{2}-\alpha_{1} i \xi v_{1}}{i \xi}$, etc., which means that all coordinates of $v$ can be calculated from a given $v_{1}$ by recurrent formulas. This implies the desired result.

Now let $v_{1}$ be an eigenvector and $v_{2}$ the first associated vector corresponding to a nonzero eigenvalue $\mu$. Let us show that none of their linear combinations can belong to the linear hull of the vectors $\left\{e_{3}, \ldots, e_{n}\right\}$. Two cases are possible: $e_{1}^{\mathrm{T}} v_{2}=0$ or $e_{1}^{\mathrm{T}} v_{2} \neq 0$. In the first case, the conditions $e_{j}^{\mathrm{T}}\left(c_{1} v_{1}+c_{2} v_{2}\right)=0, j=1,2$, 
together with the fact that $v_{1}$ is an eigenvector, immediately imply that $c_{1}=c_{2}=0$. If $e_{1}^{\mathrm{T}} v_{2} \neq 0$, then, similarly to the case of two eigenvalues, we obtain a system of equations for $c_{1}, c_{2}$ :

$$
\begin{gathered}
\left(e_{1}^{\mathrm{T}} v_{1}\right) c_{1}+\left(e_{1}^{\mathrm{T}} v_{2}\right) c_{2}=0, \\
(\mu+1)\left(e_{1}^{\mathrm{T}} v_{1}\right) c_{1}+\mu\left(e_{1}^{\mathrm{T}} v_{2}\right) c_{2}=0,
\end{gathered}
$$

which obviously has a nonzero determinant. Therefore, $c_{1}=c_{2}=0$.

Going back to the projection problem, we conclude from the above that for all $\xi \neq 0$ there is an eigenspace $V=\operatorname{Lin}\left\{v_{1}, v_{2}\right\}$ that satisfies the necessary and sufficient conditions for the existence of a solution, which means that the projection into two equations exists for all $\xi$.

It should be mentioned that our investigation of the quadratic matrix equation itself was not the only purpose of this work. Studying dynamics separation conditions for linearized moment approximations of kinetic equations entails matrix equations algebraically depending on a parameter $\xi$, the dual of $x$ under the Fourier transformation. One has to deal with a much more difficult problem: to establish the existence of a solution of a matrix equation continuously depending on $\xi$. Thus, for the above example with odd $n$, taking into account the continuity of solutions of the parametric matrix equation makes it necessary to single out the so-called windows of the parameter $q$ for which there exists only one of the possible projections, $n \rightarrow 1$ or $n \rightarrow n-1$. Conditions of the existence of continuous solutions of parametric matrix equations will be studied in another publication.

\section{REFERENCES}

1. Chen Gui-Qiang, C. D. Levermore, and Tai-Ping Luui, "Hyperbolic conservation laws with stiff relaxation terms and entropy," Commun. Pure Appl. Math., 47, 787-830 (1994).

2. V. A. Palin and E. V. Radkevich, "Navier-Stokes approximation and Chapman-Enskog projection problems for kinetic equations," Tr. Semin. Petrovskogo, 25, 184-225 (2006).

3. S. I. Gelfand, "On the number of solutions of a quadratic equation," in: GLOBUS, Mathematics Seminar, Vol. 1 [in Russian], Moscow Center of Continuous Mathematical Education, Moscow (2004), pp. $124-133$.

4. V. V. Kozlov, "Restrictions of quadratic forms to Lagrangian planes, quadratic matrix equations, and gyroscopic stabilization," Funkts. Anal. Prilozh., 39, 1-14 (2005).

5. Lu Tongxing, "Solution of the matrix equation $A X-X B=C$," Computing, 37, 351-355 (1986).

6. V. M. Prokip, "On the solvability of the Riccati matrix algebraic equation," Ukr. Math. J., 46, No. 11, 1763-1766 (1994).

7. G. I. Shurbet, T. O. Lewis, and T. L. Boullion, "Quadratic matrix equations," Ohio J. Sci., 74, No. 5, 273-277 (1974).

8. W. Dreyer and H. Struchtrup, "Heat pulse experiments revisted," Contin. Mech. Thermodyn., 5, 3-50 (1993).

9. C. D. Levermore, "Moment closure hierarchies for kinetic theories," J. Stat. Phys., 83, 1021-1065 (1996).

10. E. V. Radkevich, "Chapman-Enskog projections and Navier-Stokes approximation problems," $\operatorname{Tr}$. MIAN Steklova, 250, 219-225 (2005).

V. V. Palin

Moscow State University, Moscow, Russia 